\title{
An innovative wheel-rail contact model for multibody applications
}

\author{
Silvia Magheri, Monica Malvezzi, Enrico Meli*, Andrea Rindi \\ University of Florence, Department of Energy Engineering S. Stecco, Via S. Marta 3, 50139 Firenze, Italy
}

\section{A R T I C L E I N F O}

\section{Article history:}

Received 8 September 2010

Accepted 3 October 2010

Available online 12 October 2010

\section{Keywords:}

Multibody modeling

Wheel-rail contact

Contact between elastic bodies

\begin{abstract}
A B S T R A C T
In this work an innovative elastic wheel-rail contact model is presented. The model considers the wheel and the rail as elastic deformable bodies and requires numerical solution of Navier's elasticity equation. The contact between wheel and rail has been described by means of suitable analytical contact conditions. The contact model was subsequently inserted into a multibody model of a benchmark railway vehicle (the Manchester Wagon) in order to obtain a complete model of the wagon. The complete model has been implemented in the Matlab/Simulink environment. Numerical simulations of the vehicle dynamics have been carried out on many different railway tracks with the aim of evaluating the performance of the model.

The main objective of the authors is to achieve a better integration between the differential and multibody modeling. This kind of integration is almost absent in the literature (especially in the railway field) due to the computational cost and to the memory consumption. It is, however, very important as only differential modeling allows accurate analysis of the contact problem (in terms of contact forces, position and shape of the contact patch, stresses and strains), while multibody modeling is generally accepted as the current standard for studying railway dynamics.
\end{abstract}

(C) 2010 Elsevier B.V. All rights reserved.

\section{Introduction}

The multibody simulation of railway vehicle dynamics needs a reliable contact model that satisfies the following specifics: accurate description of the global and local contact phenomena (contact forces, position and shape of the contact patch, stresses and strains), general and robust handling of the multiple contact, high numerical efficiency and compatibility with commercial multibody software (Simpack Rail, Adams Rail).

The wheel-rail contact problem has been discussed by several authors and many models can be found in the literature. All the contact models specifically designed for the multibody modeling (as the so-called rigid contact formulation [1-6] and the semi-elastic contact description [4-8]) are computationally very efficient but their generality and accuracy is often insufficient. In particular, the physical theories behind these kinds of models (Hertz's and Kalker's theory) require very restrictive hypotheses that, in many circumstances, are unverified.

Differential contact models are needed if a detailed description of the contact phenomena is required. In other words, wheel and rail have to be considered as elastic bodies governed by the

\footnotetext{
* Corresponding author. Fax: +390554796342.

E-mail addresses: silvia.magheri@unifi.it (S. Magheri), malvezzi@mapp1.de.unifi.it (M. Malvezzi), meli@mapp1.de.unifi.it (E. Meli), rindi@mapp1.de.unifi.it (A. Rindi).
}

Navier's equations and the contact has to be described by suitable analytical contact conditions. This kind of approach assures high generality and accuracy but at a higher computational cost and memory consumption [4,9-13]. For this reason, the integration between multibody and differential modeling is almost absent in the literature, especially in the railway field. However, this integration is very important as only differential modeling allows an accurate analysis of the contact problem, while the multibody modeling is generally accepted as the standard for studying railway dynamics.

In this work an innovative differential contact model is presented with the aim of achieving a better integration between multibody and differential modeling. The new contact model is fully 3D and satisfies all the specifics described above. The developed procedure requires the discretization of the elastic contact problem (Navier's equations and analytical contact condition) and subsequently the solution of the nonlinear discrete problem. Both the steps have been implemented in the Matlab/Simulink environment.

At this point the contact model has been inserted into a 2D multibody model of a railway vehicle to obtain a complete model of the wagon. The railway vehicle chosen as the benchmark is the Manchester Wagon [14]. The choice of 2D multibody model allows us to study lateral vehicle dynamics while reducing computational load. In the near future fully 3D multibody models will be considered in order to have a complete description of vehicle dynamics. The multibody model has been implemented in 

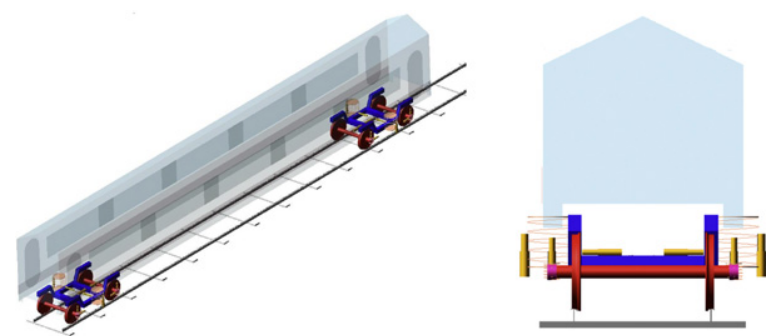

Fig. 1. 3D and 2D multibody models of the Manchester Wagon.

SimMechanics, which is a Matlab toolbox specifically designed for multibody dynamics.

A 2D multibody model of the same vehicle (this time equipped with a standard contact model based on the semi-elastic approach) was then assessed using Simpack Rail, which is a widely tested and validated commercially available multibody software used for modeling railway vehicle behaviour.

Finally numerical simulations of the vehicle dynamics have been carried out on many different railway tracks with the aim of evaluating the performance of the whole model. The comparison between the results obtained by the Matlab model and those obtained by the Simpack Rail model has allowed an accurate and reliable validation of the new contact model.

\section{Architecture of the model}

As noted in Section 1, the whole model consists of two parts; the 2D multibody model of the railway vehicle and the 3D differential wheel-rail contact model. The 2D model has been obtained from a fully 3D multibody model of the benchmark vehicle (the Manchester Wagon, Fig. 1).

The 2D model consists of three bodies; a car-body, a bogie and a wheelset. The car-body and the bogie have 3 degrees of freedom (lateral and vertical displacement and roll) while the wheelset has 4 degrees of freedom (lateral and vertical displacement, roll and pitch, i.e. the rotation around its symmetry axis). In other words the wheelset has been considered as a 3D body.

During simulation the 2D multibody model interacts with the fully 3D differential contact model. The general architecture of the model is schematically shown in Fig. 2.

At each integration step the multibody model evaluates the kinematic variables relative to the wheelset (position, orientation and their derivatives). Starting from these quantities, the contact model calculates the global and local contact variables (forces, contact patch, stresses and displacements). Finally the calculated contact variables allow the multibody model to simulate vehicle dynamics.

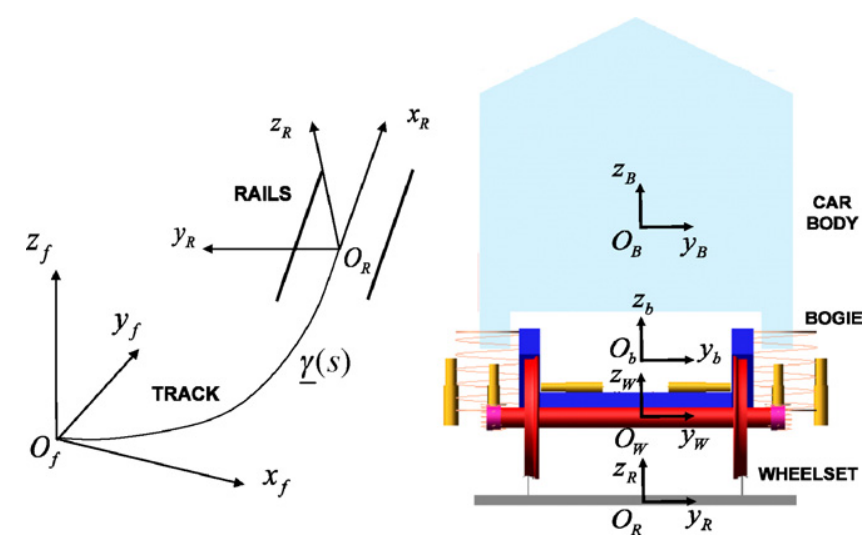

Fig. 3. Reference systems relative to the multibody model.

\section{Reference systems}

The railway track can be considered as a 3D curve $\gamma(s)$ expressed in a fixed reference system $O_{f} x_{f} y_{f} z_{f}$ (where $s$ is the curvilinear abscissa of $\gamma$ ). Usually in the cartographic description of the track only the curvature $K(s)$ of $\gamma(s)$ and the track slope $p(s)$ are known; however, the knowledge of these parameters is enough to rebuild the curve $\gamma(s)[8,15]$.

In this work the lateral vehicle dynamics will be described in a local reference system $O_{R} x_{R} y_{R} z_{R}$ having the $x_{R}$ axis tangent to the track in the point $O_{R}=\gamma(s)$ and the $z_{R}$ axis normal to the plane of the rails. In the considered case the time histories of the curvilinear abscissa $s(t)$ and of the origin $O_{R}=\gamma(s(t))$ are supposed to be known (for instance they can be calculate $\bar{d}$ by simulating the longitudinal vehicle dynamics independently).

The local system follows the motion of the whole model along the track so that the centers of mass of the bodies lie always on the plane $y_{R} z_{R}$. As noted in Section 2, the car-body and the bogie can only translate along $y_{R}$ and $z_{R}$ and rotate around $x_{R}$ while the wheelset can also rotate around its symmetry axis.

Subsequently a third reference system $O_{W} x_{W} y_{W} z_{W}$ is defined. The origin $O_{W}$ coincides with the center of mass of the wheelset and the $y_{W}$ axis with its symmetry axis. This system is fixed to the wheelset except for the rotation around the axis. Finally two reference systems $O_{b} x_{b} y_{b} z_{b}$ and $O_{B} x_{B} y_{B} z_{B}$ are introduced, fixed, respectively, to the bogie and to the car-body. As usual the origins coincide with the centers of mass. The placement of the reference systems is illustrated in Fig. 3.

In order to correctly describe the differential contact model, two further reference systems have to be defined for each wheel-rail pair. For the sake of simplicity only the left pair has been reported in Fig. 4. The first system $O_{l w} x_{l w} y_{l w} z_{l w}$ is parallel to the system

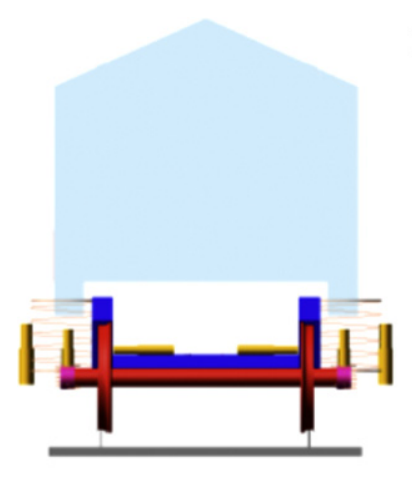

Kinematic Variables
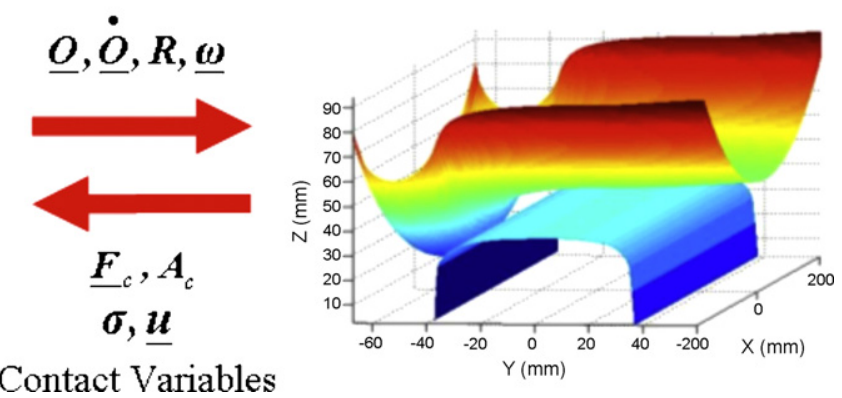

Fig. 2. General architecture of the model. 


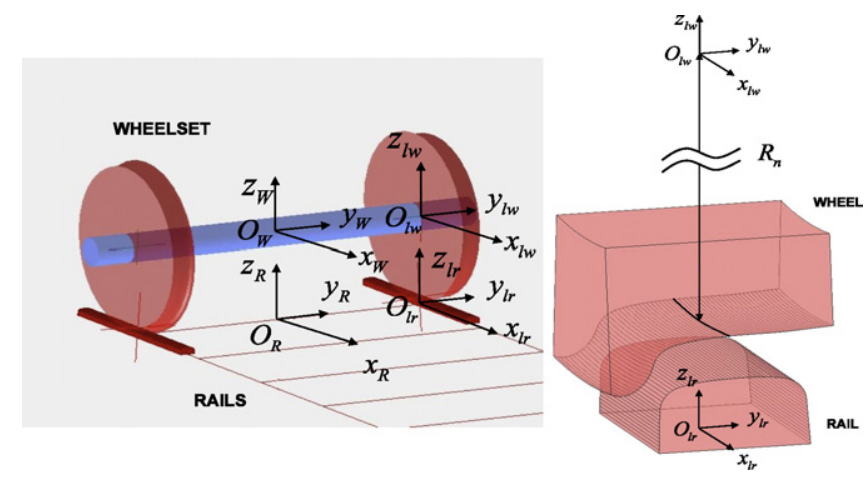

Fig. 4. Reference systems relative to the differential contact model.

$O_{W} x_{W} y_{W} z_{W}$ and its origin $O_{l w}$ lies on the symmetry axis of the wheel. The system is fixed to the wheel except for the rotation around the $y_{l w}$ axis.

Moreover, the origin $O_{l w}$ belongs to the nominal rolling plane, i.e. the plane normal to the rotation axis containing the nominal rolling radius. The second system $O_{l r} x_{l r} y_{l r} z_{l r}$ is parallel to the system $O_{R} x_{R} y_{R} z_{R}$. Its origin $O_{l r}$ belongs to the axis $y_{R}$ while the distance between $O_{R}$ and $O_{l r}$ has to assure the correct gauge between the rails. Both the reference systems described above are very important because the global and local contact variables will be evaluated by the contact model in these systems only.

Finally, in regards to the external forces acting on the bodies, some considerations are needed. As noted previously, the lateral vehicle dynamics is studied in the local reference system $O_{R} x_{R} y_{R} z_{R}$, however, this system is not inertial. Therefore, the multibody model will have to consider the effect of the fictitious forces (centrifugal force and Coriolis force). These quantities can be calculated from the kinematics of the bodies as a function of the curvature $K(s)$ and of the track slope $p(s)$. [15]

\section{The 2D multibody model}

The 2D multibody model has been obtained from a fully 3D multibody model of the Manchester Wagon. The physical and geometrical characteristics of which are easily available in the literature [14] The original 3D model consists of:

- 1 car-body, 2 bogies and 4 wheelsets;

- rear and front primary suspensions;

- rear and front secondary suspensions (including roll bar, traction rod and bumpstop).

Both the primary and the secondary suspensions are usually modeled by means of nonlinear force elements such as threedimensional springs and dampers. The $2 \mathrm{D}$ model can be thought of as a section of the 3D model and comprises (Fig. 5):

- one car-body, one bogie and one wheelset;

- one primary suspension;

- one secondary suspension (including roll bar and bumpstop).

It should be noted that only some degrees of freedom (DOF) are allowed by the 2D model:

- the car-body and the bogie have 3 DOF; they can translate along the axes $y_{R}$ and $z_{R}$ (lateral and vertical displacements) and rotate around the $x_{R}$ axis (roll);

- the wheelset, considered as a 3D body, has 4 DOF; where in addition to the previous DOF it can rotate around its symmetry axis $y_{W}$ (pitch).

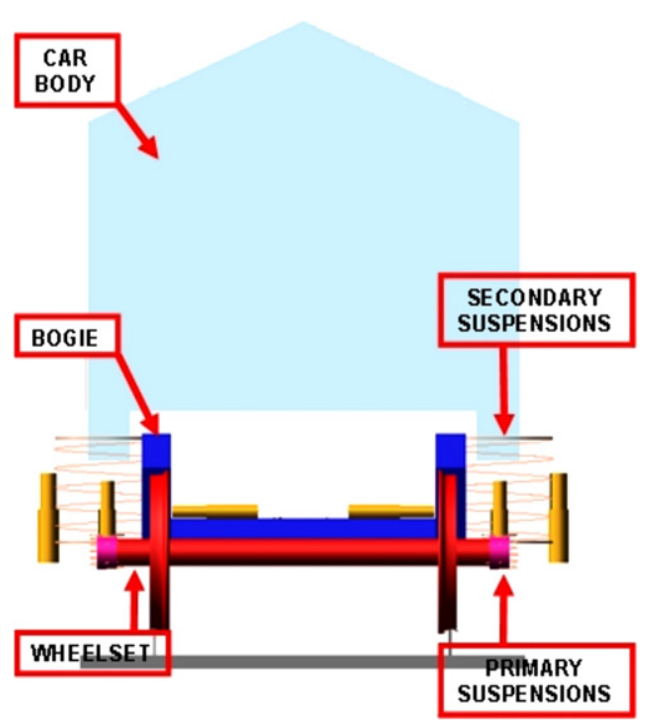

Fig. 5. 2D multibody model of the Manchester Wagon.

Moreover, in order to assure the dynamic equivalence between the 2D model and the original 3D model, the inertial characteristics of the bodies and the physical characteristics of the force elements have to be correctly scaled down $[5,14]$. These scaling factors are schematically reported in Tables 1 and 2 .

The choice of a 2D multibody model has been made with the aim of studying lateral vehicle dynamics and, at the same time, reducing the computational load. In the near future fully 3D multibody models of the Manchester Wagon will be considered in order to have a complete description of the vehicle dynamics.

\section{The 3D differential contact model}

In regards to the generic contact variable $Z$, the following convention will be adopted:

- $Z_{w 0}$ and $Z_{w}^{r}$ will denote a variable relative to the wheel, respectively, expressed in the reference systems $O_{l w} x_{l w} y_{l w} z_{l w}$ and $O_{l r} x_{l r} y_{l r} z_{l r}$

- $Z_{r}$ and $Z_{r}^{w}$ will denote a variable relative to the rail, respectively, expressed in the reference systems $O_{l r} x_{l r} y_{l r} z_{l r}$ and $O_{l w} x_{l w} y_{l w} z_{l w}$.

\subsection{Inputs and outputs}

With reference to Fig. 2, the contact model can be thought of as a black box having the following inputs and outputs:

Table 1

Scaling factors (mass and inertia).

\begin{tabular}{lll}
\hline Body & Mass & Inertia \\
\hline Car-body & 0.25 & 0.25 \\
Bogie & 0.5 & 0.5 \\
Wheelset & 1 & 1 \\
\hline
\end{tabular}

Table 2

Scaling factors (springs and dampers).

\begin{tabular}{lll}
\hline Suspensions & Springs & Dampers \\
\hline Secondary & 0.5 & 0.5 \\
Primary & 1 & 1 \\
\hline
\end{tabular}



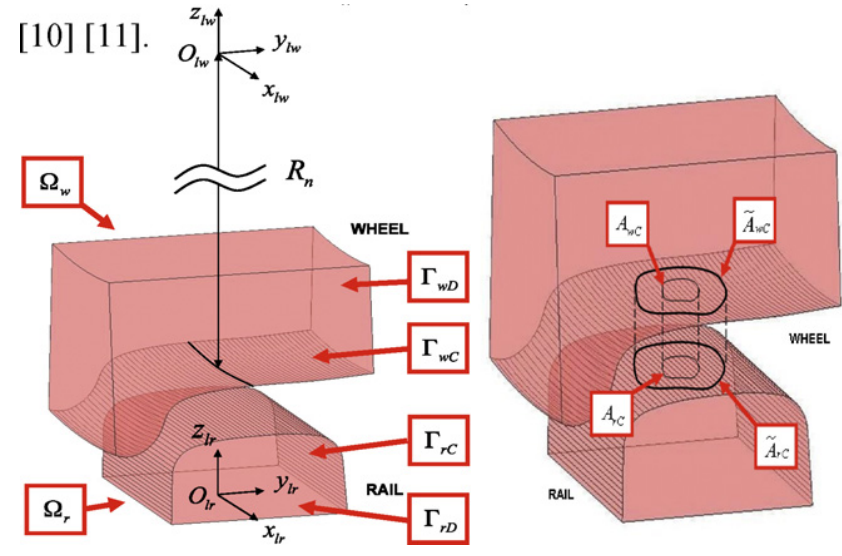

Fig. 6. The problem geometry.

- Inputs: the kinematic variables relative to the considered wheel-rail pair (in this case the left one), i.e. the position $\underline{O}_{w}^{r}$, the velocity $\underline{\dot{O}}_{w}^{r}$, the orientation $R_{w}^{r}$ and the angular velocity $\underline{\omega}_{w}^{r}$ of the reference system $O_{l w} x_{l w} y_{l w} z_{l w}$ with respect to the system $O_{l r} x_{l r} y_{l r} z_{l r}$ (see Fig. 4).

- Outputs: the global and local contact variables relative to the wheel and to the rail, like the contact forces $\underline{F}_{w C}$ and $\underline{F}_{r C}$, the stresses $\sigma_{w}$ and $\sigma_{r}$, the displacements $\underline{u}_{w}$ and $\underline{u}_{r}$ and the contact patches $A_{w C}$ and $A_{r C}$.

\subsection{The kinematics of the problem}

The wheel and the rail have been considered as two linear elastic bodies $\Omega_{w}$ and $\Omega_{r}$ (as shown in Fig. 6) [10,11].

Both the domains are supposed to be large enough with respect to the dimensions of the contact patch. The boundaries $\partial \Omega_{w}$ and $\partial \Omega_{r}$ are split into two disjointed regions $\Gamma_{w D}, \Gamma_{w C}$ and $\Gamma_{r D}, \Gamma_{r C}$, respectively. Within the regions $\Gamma_{w D}$ and $\Gamma_{r D}$ the displacements are fixed (and equal to zero) while $\Gamma_{w c}$ and $\Gamma_{r c}$ (dashed in the figure) are the regions where contact may occur. In the case of contact the geometric intersection between the surfaces $\Gamma_{w c}$ and $\Gamma_{r C}$ (and thus between the non-deformed configurations) allows us to define two regions $\widetilde{A}_{w C} \subset \Gamma_{w C}$ and $\widetilde{A}_{r C} \subset \Gamma_{r C}$ (with $\widetilde{A}_{w C} \simeq \widetilde{A}_{r C}$ ) that can be considered as a rough estimate of the contact area. The situation is schematically sketched in Figs. 6 and 7.

The real contact areas $A_{w C} \subset \widetilde{A}_{w C}$ and $A_{r C} \subset \widetilde{A}_{r C}$ (with $A_{w C} \simeq A_{r C}$ ) are unknown and have to be calculated by the model. For this purpose a contact map $\Phi$ has to be introduced. The contact map $\underline{\Phi}: \widetilde{A}_{w C} \rightarrow \widetilde{A}_{r C}$ (by convention the wheel is the master body) locates
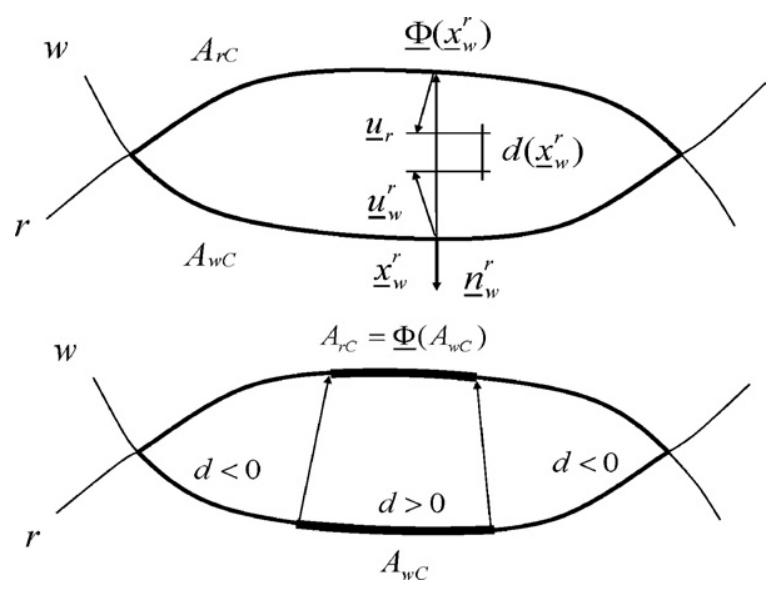

Fig. 7. Contact map and distance function. the point on the rail $\underline{\Phi}\left(\underline{x}_{w}^{r}\right) \in \widetilde{A}_{r C}$ that makes contact with the generic point on the wheel $\underline{x}_{w}^{r} \in \widetilde{A}_{w C}$ in the deformed configuration.

In this case the map $\underline{\Phi}$ is defined as the normal projection $\underline{\Phi}\left(\underline{x}_{w}^{r}\right)$ of the point $\underline{x}_{w}^{r} \in \widetilde{A}_{w C}$ on the surface $\widetilde{A}_{r C}$.

Starting from the contact map, the distance function between the deformed configurations $d: \widetilde{A}_{w C} \rightarrow R$ can be evaluated:

$d\left(\underline{x}_{w}^{r}\right)=\left(\underline{u}_{w}^{r}-\underline{u}_{r}\right) \cdot \underline{n}_{w}^{r}-\left(\underline{\Phi}\left(\underline{x}_{w}^{r}\right)-\underline{x}_{w}^{r}\right) \cdot \underline{n}_{w}^{r}$

where $\underline{n}_{w}^{r}$ is the outgoing normal versor to the surfaces $\Gamma_{w c}$. The function $d$ is positive if there is penetration between the deformed configurations and negative otherwise.

Formally the contact area $A_{w C}$ is defined as the region of $\widetilde{A}_{w C}$, where the function $d$ is positive while the contact area $A_{r C}=\underline{\Phi}\left(A_{w C}\right)$ is the normal projection of $A_{w C}$ on $\widetilde{A}_{r C}$. In other words, from a kinematic point of view, penetration between the deformed bodies is allowed and will play a fundamental role in the contact model (see Section 5.3) [10,11].

In this way the estimated contact areas $\widetilde{A}_{w C}$ and $\widetilde{A}_{r C}$ depend only on the relative wheel-rail kinematics $\left(\underline{O}_{w}^{r}, \underline{\dot{O}}_{w}^{r}, R_{w}^{r}\right.$ and $\left.\underline{\omega}_{w}^{r}\right)$ while the real contact areas $A_{w C}$ and $A_{r C}$ depend also on the displacements $\underline{u}_{w}$ and $\underline{u}_{r}$. Finally it is useful to note that no hypothesis has been made on the shape of the contact patch; in particular, the contact patch can be made up of one or more disjoint parts.

In regards to the wheel and rail profiles, the standard ORE S 1002 and UIC 60 have been used [15].

\subsection{The contact model}

According to the linear theory of elasticity $[10,11]$, both the wheel and the rail are governed by Navier's equations:

$$
\begin{array}{llll}
\operatorname{div} \sigma_{w}\left(\underline{u}_{w}\right)=\underline{0} & \text { on } \Omega_{w} & \sigma_{w}\left(\underline{u}_{w}\right) \underline{n}_{w}=\underline{p}_{w} & \text { on } \widetilde{\mathrm{A}}_{w C} \\
\operatorname{div} \sigma_{r}\left(\underline{u}_{r}\right)=\underline{0} & \text { on } \Omega_{r} & \sigma_{w}\left(\underline{u}_{w}\right) \underline{n}_{w}=\underline{0} & \text { on } \Gamma_{w C} \backslash \widetilde{\mathrm{A}}_{w C} \\
\underline{u}_{w}=\underline{0} & \text { on } \Gamma_{w D} & \sigma_{r}\left(\underline{u}_{r}\right) \underline{n}_{r}=\underline{p}_{r} & \text { on } \widetilde{\mathrm{A}}_{r C} \\
\underline{u}_{r}=\underline{0} & \text { on } \Gamma_{r D} & \sigma_{r}\left(\underline{u}_{r}\right) \underline{n}_{r}=\underline{0} & \text { on } \Gamma_{r C} \backslash \widetilde{\mathrm{A}}_{r C}
\end{array}
$$

where $\underline{n}_{w}$ and $\underline{n}_{r}$ are the outgoing normal vectors to the surfaces $\Gamma_{w C}$ and $\Gamma_{r C}$ while $\underline{p}_{w}$ and $\underline{p}_{r}$ are the unknown contact pressures. The pressures $\underline{p}_{w}$ and $\underline{p}_{r}$ are defined on $\widetilde{A}_{w C}$ and $\widetilde{A}_{r C}$ but, according to Section 5.2, will have to be zero on $\widetilde{A}_{w C} \backslash A_{w C}$ and $\widetilde{A}_{r C} \backslash A_{r C}$. Both the bodies have the material characteristics of the steel (Young's modulus $E_{w}=E_{r}=2.1 \times 10^{11}$ Pa and Poisson's coefficient $v_{w}=$ $v_{r}=0.3$ ). In the studied case the volume forces (i.e. the gravity) have been neglected because the multibody model of the wheelset already considers their effect. Moreover, since the solution is supposed to be steady within the integration step (see Fig. 2), the inertial terms have also been omitted.

Similarly the problem (2) can be formulated in a weak form, as follows:

$$
\begin{array}{ll}
\int_{\Omega_{w}} \sigma_{w}\left(\underline{u}_{w}\right): \varepsilon_{w}\left(\underline{v}_{w}\right) d V=\int_{\tilde{A}_{w C}} \underline{p}_{w} \cdot \underline{v}_{w} d A & \forall \underline{v}_{w} \in V_{w} \\
\int_{\Omega_{r}} \sigma_{r}\left(\underline{u}_{r}\right): \varepsilon_{r}\left(\underline{v}_{r}\right) d V=\int_{\tilde{A}_{r C}} \underline{p}_{r} \cdot \underline{v}_{r} d A & \forall \underline{v}_{r} \in V_{r}
\end{array}
$$

where $\varepsilon_{w}$ and $\varepsilon_{r}$ are the strains while $V_{w}$ and $V_{r}$ are suitable Sobolev's spaces.

In order to complete the contact model, the contact pressures $\underline{p}_{w}$ and $\underline{p}_{r}$ have to be expressed as a function of the displacements $\underline{u}_{w}$ and $\underline{u}_{r}$.

For the sake of simplicity the normal and the tangential contact pressures on the wheel are introduced: $p_{w N}^{r}=\underline{p}_{w}^{r} \cdot \underline{n}_{w}^{r}, \underline{p}_{w T}^{r}=\underline{p}_{w}^{r}-$ $p_{w N}^{r} \underline{n}_{w}^{r}$. 

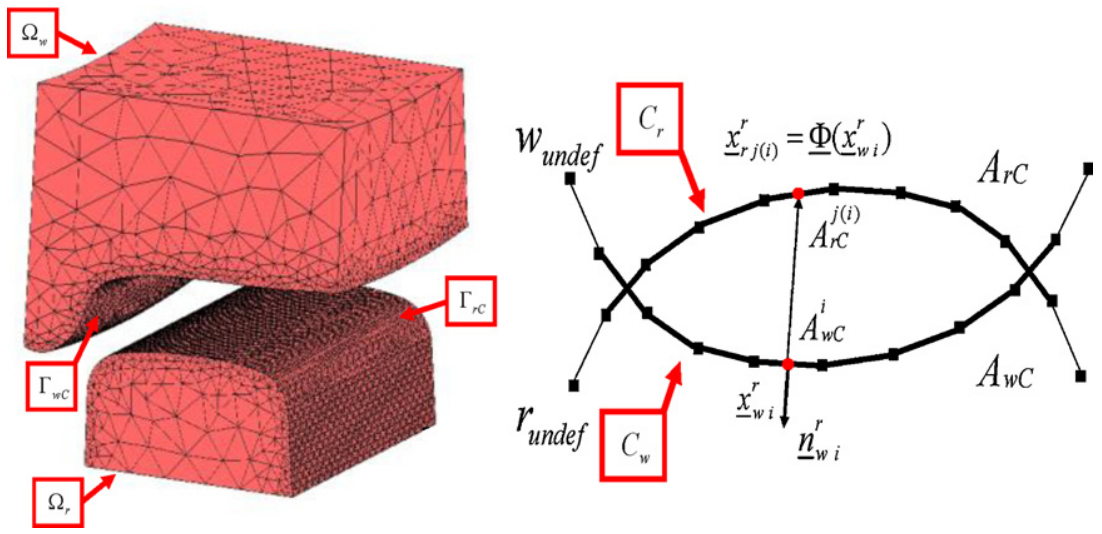

Fig. 8. Discretization of the contact model.

The normal pressure $p_{w N}^{r}$ has been calculated by means of the distance function $d$ :

$p_{w N}^{r}\left(\underline{x}_{w}^{r}\right)=-K \max \left(d\left(\underline{x}_{w}^{r}\right), 0\right)$ on $\widetilde{A}_{w C}$

where $K>0$ is a fictitious stiffness constant. The value of $K$ has to be large enough to assure the accuracy required by these problems. The condition of ideal contact (total absence of penetration between the deformed bodies) is reached for $K \rightarrow+\infty$ (usually $K \geq 10^{15} \mathrm{~N} / \mathrm{m}^{3}$ ) [10,11].

To evaluate the tangential pressure $\underline{p}_{w T}^{r}$, the slip $\underline{s}_{w}^{r}$ between the wheel and rail surfaces has to be defined. Since the solution is supposed to be steady within the integration step, the following expression holds [4]:

$$
\begin{aligned}
\underline{s}_{w}^{r}\left(\underline{x}_{w}^{r}\right)= & \underline{w}_{w}^{r}\left(\underline{x}_{w}^{r}\right)+\underline{u}_{w}^{r}\left(\underline{x}_{w}^{r}\right)-\underline{w}_{r}\left(\underline{\Phi}\left(\underline{x}_{w}^{r}\right)\right)-\underline{\dot{u}}_{r}\left(\underline{\Phi}\left(\underline{x}_{w}^{r}\right)\right) \\
= & \underline{w}_{w}^{r}\left(\underline{x}_{w}^{r}\right)+J_{w}^{r}\left(\underline{x}_{w}^{r}\right) \underline{w}_{w}^{r}\left(\underline{x}_{w}^{r}\right)-\underline{w}_{r}\left(\underline{\Phi}\left(\underline{x}_{w}^{r}\right)\right) \\
& -J_{r}\left(\underline{\Phi}\left(\underline{x}_{w}^{r}\right)\right) \underline{w}_{r}\left(\underline{\Phi}\left(\underline{x}_{w}^{r}\right)\right)
\end{aligned}
$$

where $\underline{w}_{w}^{r}$ and $\underline{w}_{r}$ are the rigid velocity of the points $\underline{x}_{w}^{r}$ and $\underline{\Phi}\left(\underline{x}_{w}^{r}\right)$ while $J_{w}^{r}$ and $J_{r}$ are the Jacobians of $\underline{u}_{w}^{r}$ and $\underline{u}_{r}$. As usual the normal and the tangential slips are: $s_{w N}^{r}=\underline{s}_{w}^{r} \cdot \underline{n}_{w}^{r}, \underline{s}_{w T}^{r}=\underline{s}_{w}^{r}-s_{w N}^{r} \underline{n}_{w}^{r}$. According to the standard friction models, the tangential pressures $\underline{p}_{w T}^{r}=\underline{p}_{w T}^{r}\left(\underline{x}_{w}^{r}\right)$ can be expressed as follows:

$\underline{p}_{w T}^{r}=\left\{\begin{array}{ll}\underline{0} & \text { if } s_{w T}^{r}=0 \\ -\mu\left(s_{w T}^{r}, V\right)\left|p_{w N}^{r}\right| \frac{s_{w T}^{r}}{s_{w T}^{r}} & \text { if } s_{w T}^{r}>0\end{array}\right.$ on $\widetilde{A}_{w C}$

where $s_{w T}^{r}$ is the norm of $\underline{s}_{w T}^{r}=\underline{s}_{w T}^{r}\left(\underline{x}_{w}^{r}\right)$ and $V$ is the longitudinal velocity of the vehicle. Further details on the friction function $\mu\left(s_{w T}^{r}, V\right)$ can be found in the literature [17].

Finally the action-reaction principle (Newton's third law) allows calculation of the pressure $\underline{p}_{r}$ :

$\underline{p}_{r}\left(\underline{\Phi}\left(\underline{x}_{w}^{r}\right)\right)=-\underline{p}_{w}^{r}\left(\underline{x}_{w}^{r}\right)$ on $\widetilde{A}_{w C}$

It is useful to note that, according to the described model, the pressures $\underline{p}_{w}^{r}$ and $\underline{p}_{r}$ are zero, respectively, on $\widetilde{A}_{w C} \backslash A_{w C}$ and $\widetilde{A}_{r C} \backslash A_{r C}$.

The displacements $\underline{u}_{w}$ and $\underline{u}_{r}$ are evaluated through the numerical solution of Eq. (3). The calculation of these quantities will allow calculation of all other outputs such as the contact areas $A_{w C}$ and $A_{r C}$ and the stresses $\sigma_{w}$ and $\sigma_{r}$. The contact forces $\underline{F}_{w C}$ and $\underline{F}_{r C}$ will be estimated by integration:

$\underline{F}_{w C}=\int_{\widetilde{A}_{w C}} \underline{p}_{w} d A \quad \underline{F}_{r C}=\int_{\widetilde{A}_{r C}} \underline{p}_{r} d A$

\subsection{The discretization of the model}

Both the elastic bodies have been discretized by means of tetrahedral elements and linear shape functions. The meshes have been built according to standard Delaunay's algorithms (see Fig. 8) [16].

The resolution of the meshes on the surfaces $\Gamma_{w c}$ and $\Gamma_{r c}$ is uniform (usually in the range $1-2 \mathrm{~mm}$ ) because the position and the dimensions of the contact patch are unknown.

The surface resolution must also to ensure an appropriate accuracy that will correctly describe the contact phenomena. Moreover, it is important to note that the meshes have been built directly in the reference systems $O_{l w} x_{l w} y_{l w} z_{l w}$ and $O_{l r} x_{l r} y_{l r} z_{l r}$; therefore they do not change during the simulation and can be easily built offline.

In the future the following convention will be adopted:

- The sets of all the elements of wheel and rail will be called $T_{w}$ and $T_{r}$ while the vectors $\underline{u}_{w h}, \underline{u}_{r l} \in R^{12}$ will contain the displacements of the four nodes belonging to the elements $h \in T_{w}$ and $l \in T_{r}$. Finally the vectors $\underline{U}_{w}$ and $\underline{U}_{r}$ will comprise the displacements relative to all the nodes of wheel and rail. Since the displacements on $\Gamma_{w D}$ and $\Gamma_{r D}$ are zero, the dimension of $\underline{U}_{w}, \underline{U}_{r}$ are $3\left(N_{w}-N_{w D}\right)$ and $3\left(N_{r}-N_{r D}\right)$, where $N_{w}$ and $N_{r}$ are the numbers of nodes of wheel and rail while $N_{w D}$ and $N_{r D}$ are the numbers of nodes on $\Gamma_{w D}$ and $\Gamma_{r D}$.

- Similarly, $C_{w}$ and $C_{r}$ will be the sets of the active contact elements on wheel and on rail, i.e. the sets of the elements having, respectively, a face $\widetilde{A}_{w C}^{i}$ and $\widetilde{A}_{r C}^{j}$ that lies on $\widetilde{A}_{w C}$ and $\widetilde{A}_{r C}$. The vectors $\underline{u}_{w i}, \underline{u}_{r j} \in R^{12}$ will contain the displacements of the four nodes belonging to the elements $i \in C_{w}$ and $j \in C_{r}$ while the vectors $\underline{U}_{w C}$ and $\underline{U}_{r C}$ will comprise the displacements relative to all the active elements. The dimension of $\underline{U}_{w C}, \underline{U}_{r C}$ are $3 N_{w C}$ and $3 N_{r C}$, where $N_{w C}$ and $N_{r C}$ are the number of nodes belonging to the active elements.

The knowledge of the relative kinematics $\left(\underline{O}_{w}^{r}, \underline{\dot{O}}_{w}^{r}, R_{w}^{r}\right.$ and $\left.\underline{\omega}_{w}^{r}\right)$ and consequently of the estimated contact areas $\widetilde{A}_{w C}$ and $\widetilde{A}_{r C}$ allows to determine the sets $C_{w}$ and $C_{r}$ of the active contact elements on the wheel and on the rail.

For each active contact element on the wheel, the center $\underline{x}_{w i}^{r}$ of the face $\widetilde{A}_{w C}^{i}$ is considered. The normal projection $\underline{x}_{r j}=\underline{\Phi}\left(\underline{x}_{w i}^{r}\right)$ of $\underline{x}_{w i}^{r}$ on $\widetilde{A}_{r C}$ will belong to the external face $\widetilde{A}_{r C}^{j}$ of the $j$ th active contact element on the rail. In particular the index $j(i)$ will be a function of the index $i$. In other words the pairs of points $\left(\underline{x}_{w}^{r}, \underline{x}_{r j(i)}\right)$ with $i \in C_{w}$ can be thought of as the discretization of the contact map $\underline{\Phi}$. The situation is schematically sketched in Fig. 8. 
The values of the displacements $\underline{u}_{w}^{r}, \underline{u}_{r}$ and of their Jacobians $J_{w}^{r}, J_{r}$ in the points $\underline{x}_{w}^{r}$ and $\underline{x}_{r j}$ are evaluated through the shape functions $[10,11,16]$.

At this point the distance function $d_{i}=d\left(\underline{x}_{w}^{r} \quad\right.$ ) and the pressure $\underline{p}_{w i}^{r}=\underline{p}_{w}^{r}\left(\underline{x}_{w i}^{r}\right)$ on the face $\widetilde{A}_{w C}^{i}$ of the active element of the wheel can be calculated by means of Eqs. (1), (4) and (6). Finally a discrete version of the action-reaction principle (Newton's third law) is needed to evaluate the pressure $\underline{p}_{r j}=\underline{p}_{r}\left(\underline{x}_{r j}\right)$ on the face $\widetilde{A}_{r C}^{j}$ of the active element of the rail:

$\left|\widetilde{A}_{r C}^{j(i)} \underline{p}_{r j(i)}=\right| \widetilde{A}_{w C}^{i} \mid \underline{p}_{w i}^{r}$

where $\left|\widetilde{A}_{w C}^{i}\right|$ and $\left|\widetilde{A}_{r C}^{j}\right|$ are the areas of the faces $\widetilde{A}_{w C}^{i}$ and $\widetilde{A}_{r C}^{j}$. Both the pressures $\underline{p}_{w i}^{r}$ and $\underline{p}_{r j}$ are supposed to be constant on $\widetilde{A}_{w C}^{i}$ and $\widetilde{A}_{r C}^{j}$.

Standard FEM techniques allow us to discretize the weak form of the contact problem [see Eq. (3)] [10,11,16]:

$$
\begin{aligned}
& \int_{\Omega_{w}} \sigma_{w}\left(\underline{u}_{w}\right): \varepsilon_{w}\left(\underline{v}_{w}\right) d V=\sum_{h \in T_{w}} \underline{u}_{w}^{T}{ }_{h} K_{w} h \underline{v}_{w} h=\underline{U}_{w}^{T} K_{w} \underline{V}_{w} \\
& \int_{\Omega_{r}} \sigma_{r}\left(\underline{u}_{r}\right): \varepsilon_{r}\left(\underline{v}_{r}\right) d V=\sum_{l \in T_{r}} \underline{u}_{r}^{T}{ }_{l} K_{r} \quad \underline{v}_{r} \quad l=\underline{U}_{r}^{T} K_{r} \underline{V}_{r} \\
& \int_{\tilde{A}_{w C}} \underline{p}_{w} \cdot \underline{v}_{w} d A=\sum_{i \in C_{w}} \underline{p}_{w}^{T}{ }_{i} M_{w} \quad \underline{v}_{w} \quad \underline{v}=\underline{F}_{w}\left(\underline{U}_{w C}, \underline{U}_{r C}\right)^{T} \underline{V}_{w} \\
& \int_{\widetilde{A}_{r C}} \underline{p}_{r} \cdot \underline{v}_{r} d A=\sum_{i \in C_{w}} \underline{p}_{r}^{T}{ }_{j(i)} M_{r} \quad j(i) \underline{v}_{r} \quad j(i)=\underline{F}_{r}\left(\underline{U}_{w C}, \underline{U}_{r C}\right)^{T} \underline{V}_{r}
\end{aligned}
$$

where $K_{w h}, K_{r l}$ are the stiffness matrices relative to the elements $h \in T_{w}, l \in T_{r}$ and $M_{w i}, M_{r j}$ depend on the shape functions. The global stiffness matrices $K_{w}$ and $K_{r}$ are symmetric, positively defined and sparse, while the vectors $\underline{F}_{w}$ and $\underline{F}_{r}$ that contain the terms due to the contact pressures are sparse. Moreover, the global stiffness matrices are evaluated directly in the reference systems $O_{l w} x_{l w} y_{l w} z_{l w}$ and $O_{l r} x_{l r} y_{l r} z_{l r}$; therefore they do not change during the simulation and can be easily built offline. Eqs. (3) and (10), combined together, give:

$$
\begin{array}{ll}
\underline{U}_{w}^{T} K_{w} \underline{V}_{w}=\underline{F}_{w}\left(\underline{U}_{w C}, \underline{U}_{r C}\right)^{T} \underline{V}_{w} & \forall \underline{V}_{w} \in R^{3\left(N_{w}-N_{w D}\right)} \\
\underline{U}_{r}^{T} K_{r} \underline{V}_{r}=\underline{F}_{r}\left(\underline{U}_{w C}, \underline{U}_{r C}\right)^{T} \underline{V}_{r} & \forall \underline{V}_{r} \in R^{3\left(N_{r}-N_{r D}\right)}
\end{array}
$$

Finally, since the matrices $K_{w}, K_{r}$ are symmetric and the vectors $\underline{V}_{w}, \underline{V}_{r}$ are arbitrary, the following nonlinear system of algebraic equations is obtained:

$K_{w} \underline{U}_{w}=\underline{F}_{w}\left(\underline{U}_{w C}, \underline{U}_{r C}\right), \quad K_{r} \underline{U}_{r}=\underline{F}_{r}\left(\underline{U}_{w C}, \underline{U}_{r C}\right)$

where as noted previously, the contact displacements $\underline{U}_{w C}, \underline{U}_{r C}$ are a subset of the displacements $\underline{U}_{w}, \underline{U}_{r}$. Eq. (12) can also be written as

$\underline{U}_{w}=H_{w} \underline{F}_{w}\left(\underline{U}_{w C}, \underline{U}_{r C}\right), \quad \underline{U}_{r}=H_{r} \underline{F}_{r}\left(\underline{U}_{w C}, \underline{U}_{r C}\right)$

where the matrices $H_{w}=K_{w}^{-1}$ and $H_{r}=K_{r}^{-1}$ are symmetric, positively defined and full (consequently their storage can require an high memory consumption). Like $K_{w}$ and $K_{r}$ they do not change during the simulation and can be calculated offline. Splitting $\underline{U}_{w}, \underline{U}_{r}$ into contact displacement $\underline{U}_{w C}, \underline{U}_{r C}$ and non-contact displacement $\underline{U}_{w N C}, \underline{U}_{w N C}$, Eq. (13) becomes:

$$
\begin{aligned}
& \left(\begin{array}{c}
\underline{U}_{w N C} \\
\underline{U}_{w C}
\end{array}\right)=\left[\begin{array}{ll}
H_{w}^{11} & H_{w}^{12} \\
H_{w}^{21} & H_{w}^{22}
\end{array}\right]\left(\begin{array}{c}
\underline{0} \\
\underline{f}_{w}\left(\underline{U}_{w C}, \underline{U}_{r C}\right)
\end{array}\right) \\
& \left(\begin{array}{c}
\underline{U}_{r N C} \\
\underline{U}_{r C}
\end{array}\right)=\left[\begin{array}{ll}
H_{r}^{11} & H_{r}^{12} \\
H_{r}^{21} & H_{r}^{22}
\end{array}\right]\left(\begin{array}{c}
\underline{0} \\
\underline{f}_{r}\left(\underline{U}_{w C}, \underline{U}_{r C}\right)
\end{array}\right)
\end{aligned}
$$

In this way the second and the fourth components of Eq. (14) are sufficient to calculate contact displacements $\underline{U}_{w C}$, and $\underline{U}_{r C}$ :

$\underline{U}_{w C}=H_{w}^{22} \underline{f}_{-w}\left(\underline{U}_{w C}, \underline{U}_{r C}\right), \quad \underline{U}_{r C}=H_{r}^{22} \underline{f}_{r}\left(\underline{U}_{w C}, \underline{U}_{r C}\right)$

The matrices $H_{w}^{22}$ and $H_{r}^{22}$ have the same properties as $H_{w}$ and $H_{r}$, however, this time their dimensions are much smaller. $H_{w}^{22}$ and $H_{r}^{22}$ change during the simulation and therefore have to be built directly online. The vectors $f_{-w}$ and $f_{r}$ are full. The remaining non-contact displacements $\underline{U}_{w N C}, \underline{U}_{w N C}$ can be evaluated by means of the first and the third components of Eq. (14).

The knowledge of the displacements $\underline{U}_{w}$ and $\underline{U}_{r}$, evaluated by solving Eq. (12) or Eq. (15), allows us to calculate all the other required outputs such as the contact areas $A_{w C}$ and $A_{r C}$ and the stresses $\sigma_{w}$ and $\sigma_{r}$. The contact forces $\underline{F}_{w C}$ and $\underline{F}_{r C}$ are estimated by numerical integration:

$\underline{F}_{w C}=\sum_{i \in C_{w}}\left|\widetilde{A}_{w C}^{i}\right| \underline{p}_{w i}, \quad \underline{F}_{r C}=\sum_{i \in C_{w}}\left|\widetilde{A}_{r C}^{j(i)}\right| \underline{p}_{r j(i)}$

\subsection{The numerical solution of the discrete problem}

In this section the numerical methods used for solving the discrete contact problem (15) are presented.

Eq. (15) is a full nonlinear system with small dimensions. In particular, the typical dimensions of $H_{w}^{22}$ and $H_{r}^{22}$ (depending on the number of active elements) are about 100-1000.

Due to the small size of the problem, a Newton-LU method has been implemented in order to solve the system (15) [21]. NewtonLU is a Newton-type method for the problem $\underline{F}(\underline{x})=\underline{0}$, where $\underline{F}$ is a generic nonlinear function. In this procedure the Gauss method is employed to solve the arising linear systems:

$F^{\prime}\left(\underline{x}_{k}\right) \underline{s}_{k}=-\underline{F}\left(\underline{x}_{k}\right)$

where $F^{\prime}(\underline{x})$ is the Jacobian matrix of $\underline{F}(\underline{x})$ and $\underline{s}_{k}$ is the Newton step. It should be noted that this approach needs the computation and the storage of the whole Jacobian at each iteration. Therefore, this procedure may be very time consuming even though the dimension is relatively small.

In order to reduce the computational load, a strategy based on a Newton-Krylov method (implemented in "matrix free" way) has been considered $[18,23]$. Newton-Krylov methods are Newtontype methods, where a Krylov method is employed to approximate the arising linear systems (17).

The Krylov method computes, at each iteration, the so-called inexact Newton step $\underline{\tilde{s}}_{k}$ which satisfies the condition:

$\left\|\underline{F}^{\prime}\left(\underline{x}_{k}\right) \underline{\tilde{s}}_{k}+\underline{F}\left(\underline{x}_{k}\right)\right\| \leq \eta_{k}\left\|\underline{F}\left(\underline{x}_{k}\right)\right\|$

where the forcing terms $\eta_{k} \in[0,1)$ are used to control the level of accuracy [18]. In regards to the considered problem, numerical experimentation showed that, among all the Krylov methods, the best iterative linear solver is the BiCGStab [19]. These kinds of numerical procedures are known as Newton-BiCGStab methods.

An interesting feature of Newton-BiCGStab methods is that they require only the action of $F^{\prime}(\underline{x})$ on a vector $\underline{v}$ but not the computation and the storage of the whole Jacobian. In this case, the product $F^{\prime}(\underline{x}) v$ can be approximated by finite differences [20]:

$F^{\prime}(\underline{x}) \underline{v} \simeq \frac{\underline{F}(\underline{x}+\varepsilon \underline{v})-\underline{F}(\underline{x})}{\varepsilon}$

where $\varepsilon>0$ is a scalar small enough. Consequently these methods are called "matrix free".

It has been observed that a small number of nonlinear iterations are needed for solving the nonlinear system (15) and that convergence is achieved in almost all cases. Consequently the choice of a less accurate solution of the Newton equations (17) turned out to 
Table 3

Data of the railway track.

\begin{tabular}{lll}
\hline Curvature & $K$ & $-1 / 1200 \mathrm{~m}^{-1}$ \\
Slope & $p$ & 0 \\
Cant & $\beta$ & $60 \mathrm{~mm}$ \\
Laying angle & $\alpha_{p}$ & $1 / 40 \mathrm{rad}$ \\
Velocity & $V$ & $45 \mathrm{~m} / \mathrm{s}$ \\
Friction coefficient & $\mu$ & 0.3 \\
\hline
\end{tabular}

Table 4

Numerical parameters.

\begin{tabular}{ll} 
Differential contact model & Eq. $(15)$ \\
RelTol/AbsTol & $10^{-8} / 10^{-6}$ \\
Nonlinear solver & Newton-BiCGStab \\
Tol/MaxitNonlin & $10^{-8} / 20$ \\
$\eta /$ MaxitLin & $0.01 / 20$ \\
\hline
\end{tabular}

be very efficient and effective in reducing the norm of $\underline{F}$. A constant forcing term $\eta_{k}=\eta \leq 0.5 \forall k$ has been chosen.

Moreover, it should be noted that, if the guarantee of convergence is only local, both the numerical procedures presented may fail to find a solution - even though an effective solution exists. Therefore, Newton-LU and Newton-BiCGStab methods have been embedded into a globalization strategy. A monotone line search method and Armijo rule was employed with a maximum of 10 backtracks for nonlinear iteration [20,21].

Both the methods stop if the following stopping criterion is satisfied:

$\|\underline{F}(\underline{x})\|<$ Tol.

A comparison between the performance of the different strategies will be reported in following section.

In regards to the time integration of the whole model (multibody model and contact model; see Fig. 2), explicit ODE solvers with variable step and variable order have been considered [22]. Moreover, during the simulations, the initial conditions for the nonlinear solvers (i.e. the Newton-BiCGStab and Newton-LU methods) are continually updated in order to speed up the convergence of the solvers and to reduce the computation time. In other words the solution of the problem at the current time step is used as an initial condition for the solver at the next time step.

\section{Numerical simulation}

In order to study the behaviour of the whole model, a large number of simulations have been carried out on many different railway tracks. The performance of these models has been evaluated in terms of output accuracy (kinematic variables, contact forces and contact patch) and numerical efficiency (performance of the numerical algorithms and time consumption).

\subsection{Performance of the numerical methods}

In this section the performance of the numerical procedures described in Section 5.5 will be analyzed and compared. Using typical simulation of lateral dynamics of the Manchester Wagon $[5,15]$. The simulations have been performed on a curvilinear railway track, the data of which are reported in Table 3.

Comparison between the numerical methods has been carried out on a computer equipped with an Intel Xeon $2.66 \mathrm{GHz}$, processor, 8 GB RAM and Matlab R2007b (machine precision $\varepsilon_{m}=2 \times 10^{-16}$ ).

In order to establish the best ODE solver, several experiments were performed with the ODE23 and ODE45 solvers [22]. The value of the main numerical parameters are reported in Table 4.

RelTol and AbsTol are the relative and absolute tolerances of the ODE solvers, MaxitNonlin, MaxitLin are the maximum number of
Table 5

Comparison between ODE23 and ODE45.

\begin{tabular}{|c|c|c|c|c|}
\hline & \#cps & \#New & \#BiCGS & Time \\
\hline \multicolumn{5}{|c|}{ ODE23 - Newton-BiCGStab } \\
\hline $\mathrm{R}$ & 31,814 & 27,409 & 2.9 & $8396 \mathrm{~s}(\sim 2 \mathrm{~h} 20 \mathrm{~min})$ \\
\hline $\mathrm{L}$ & & 29,495 & 3.5 & \\
\hline \multicolumn{5}{|c|}{ ODE45 - Newton-BiCGStab } \\
\hline $\mathrm{R}$ & 45,710 & 44,625 & 3.1 & $12,870 \mathrm{~s}(\sim 3 \mathrm{~h} 34 \mathrm{~min})$ \\
\hline $\mathrm{L}$ & & 46,170 & 3.5 & \\
\hline
\end{tabular}

Table 6

Newton-LU for solving Eq. (15).

\begin{tabular}{|c|c|c|c|}
\hline & \#cps & \#New & Time \\
\hline \multicolumn{4}{|c|}{ ODE23 - Newton-LU } \\
\hline $\mathrm{R}$ & 30,401 & 23,936 & $39,096 \mathrm{~s}$ ( $\sim 10 \mathrm{~h} 51 \mathrm{~min})$ \\
\hline $\mathrm{L}$ & & 24,306 & \\
\hline
\end{tabular}

nonlinear and linear iterations, Tol is the stopping tolerance and $\eta$ is the forcing term. In particular, the value of the stopping tolerance Tol has been chosen to assure a sufficient accuracy (in terms of displacements and contact pressures) and, at the same time, to minimize the computation time.

Table 5 summarizes the results obtained using the ODE 23 and the ODE 45 solvers. For each wheel-rail contact pair (right and left) the following data have been considered.

Results include: The number \#cps of contact problem solved (equal for both the contact pairs), the total number \#New of nonlinear iterations, the average number \#BiCGS of linear iterations for each nonlinear iteration and the total computation time.

The results show that low order solvers like the ODE23 turn out to be better than high order solvers like ODE45.

As noted in Section 5.5, Eq. (15) can also be solved by means of a Newton-LU strategy. Table 6 contains the results obtained by solving (15) using this approach. The ODE solver used is the ODE23, while all other numerical parameters are the same as that reported in Table 4.

From Table 6 it can be seen that the Newton-BiCGStab methods (matrix free) are more efficient than the Newton-LU methods. In particular, the computation and the storage of the Jacobian matrix at each nonlinear iteration turned out to be too time consuming.

Finally, in order to justify the choice of the constant forcing term $\eta=10^{-2}$, experiments were performed using the following values: $\eta=0.5,10^{-1}, 10^{-2}, 10^{-3}, 10^{-4}$. The ODE solver used for these experiments was the ODE23. Results have been reported in terms of computation time (see Fig. 9).

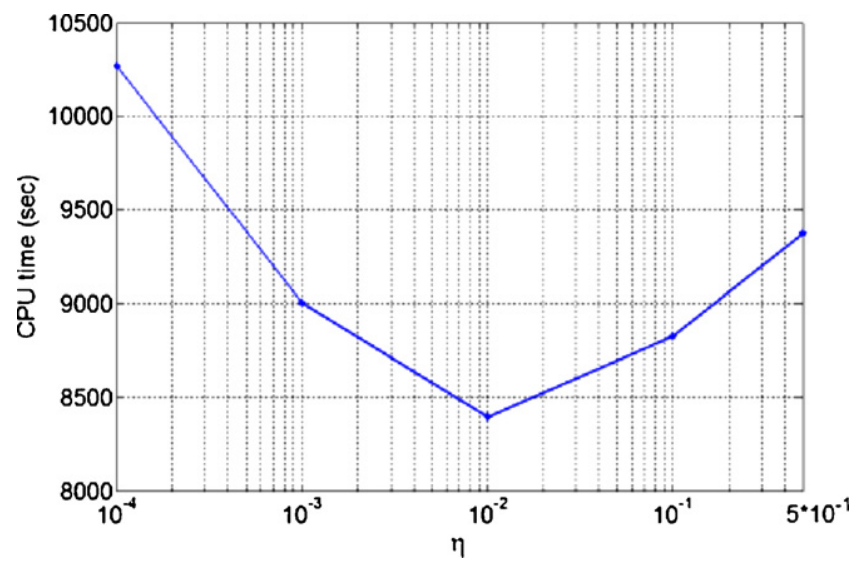

Fig. 9. Computation time as a function of the forcing term $\eta$. 

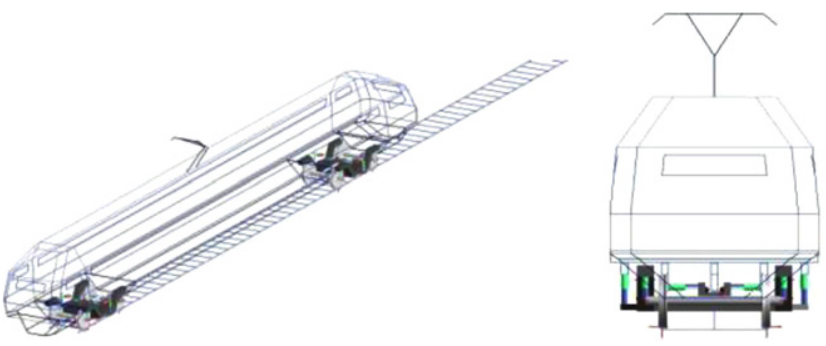

Fig. 10. 3D and 2D multibody models of the Manchester Wagon (Simpack Rail).

\subsection{The Simpack Rail 2D multibody model}

The same multibody model of the benchmark vehicle (the Manchester Wagon [14]) was also installed into Simpack Rail software for evaluation. Simpack Rail is a widely tested and validated multibody software package used for the analysis of the railway vehicle dynamics. For this assessment the multibody model is equipped with a standard contact model based on the semi-elastic approach [4-6]. As for the previous case, the 2D multibody model (designed for the study of the lateral dynamics) has been obtained from the fully 3D multibody model of the vehicle while the contact model is completely 3D (see Fig. 10). The comparison between the results obtained by the Matlab/Simulink model and those obtained by the Simpack Rail model has allowed accurate and reliable validation of the new contact model.

\subsection{Simulation of the lateral vehicle dynamics}

The comparison between the Matlab/Simulink model (on Matlab R2007b) and the Simpack Rail model (on Simpack 8.900) was carried out on the same curvilinear railway track introduced above (see Table 3) $[5,15]$. Numerical data relative to the Matlab model have been chosen starting from the results obtained in Section 6.1 (see Table 4). The ODE solver used for this simulation was the ODE23. Similarly the numerical data relative to the Simpack model are briefly summarized in Table 7.

Among all the kinematic and dynamic variables evaluated by the models, the time histories of the following quantities are reported (for the sake of simplicity all the outputs are expressed in the reference system $O_{R} x_{R} y_{R} z_{R}$ ):

- The lateral displacement $y_{W}^{R}$ of the center of mass of the wheelset $\underline{O}_{W}^{R}$ (Fig. 11).

- The lateral displacement $y_{B}^{R}$ of the center of mass of the body-car $\underline{O}_{B}^{R}$ (Fig. 12).

- The contact forces on the left wheel $\underline{F}_{l w}^{R}$ and on the right wheel $\underline{F}_{r w}^{R}$; in particular $Y_{l w}^{R}$ and $Y_{r w}^{R}$ are the lateral forces (Figs. 13 and 15) while $Q_{l w}^{R}$ and $Q_{r w}^{R}$ are the vertical forces (Figs. 14 and 16).

The Matlab variables are plotted in blue while the equivalent Simpack quantities in red (For interpretation of the references to color in the text, the reader is referred to the web version of this article).

Table 7

Numerical data (Simpack model).

\begin{tabular}{ll}
\hline ODE solver & ODE 5 (Dormand-Prince) \\
\hline Fixed step & $5 \times 10^{-4}$ \\
Contact model & Semi-elastic approach \\
\hline
\end{tabular}

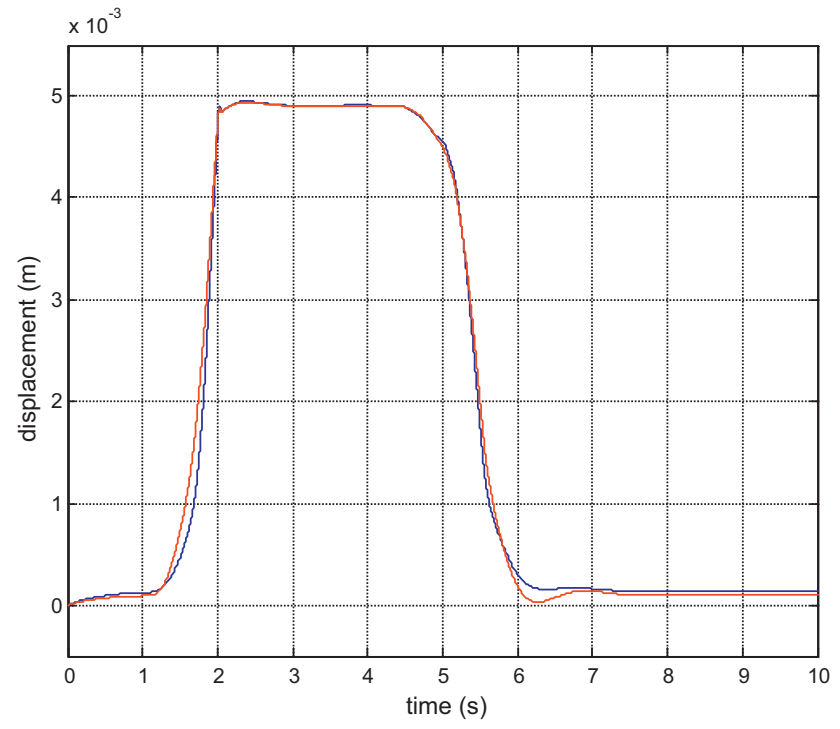

Fig. 11. Lateral displacement $y_{W}^{R}$.

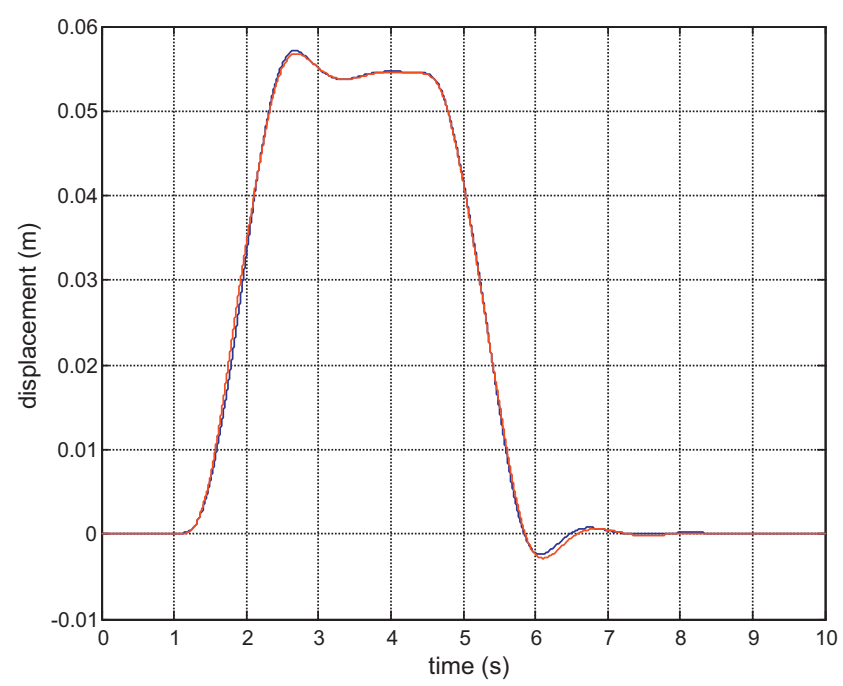

Fig. 12. Lateral displacement $y_{B}^{R}$.

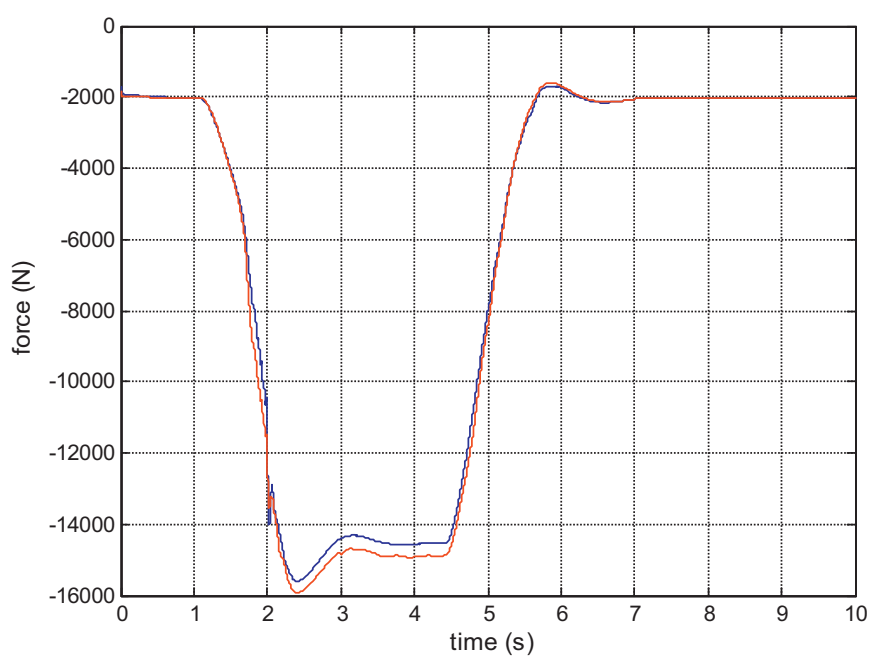

Fig. 13. Lateral force $Y_{l w}^{R}$. 


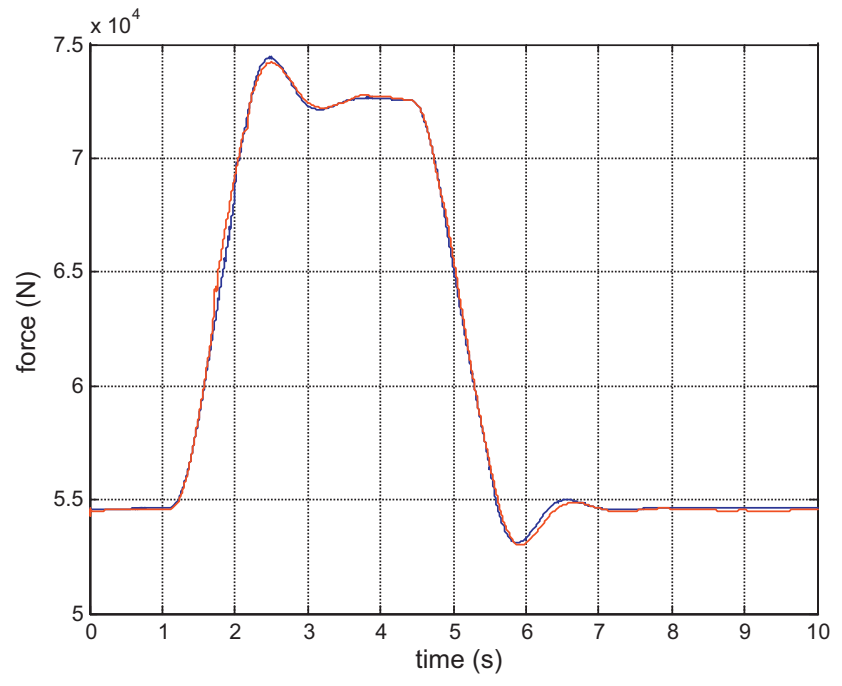

Fig. 14. Vertical force $Q_{l w}^{R}$.

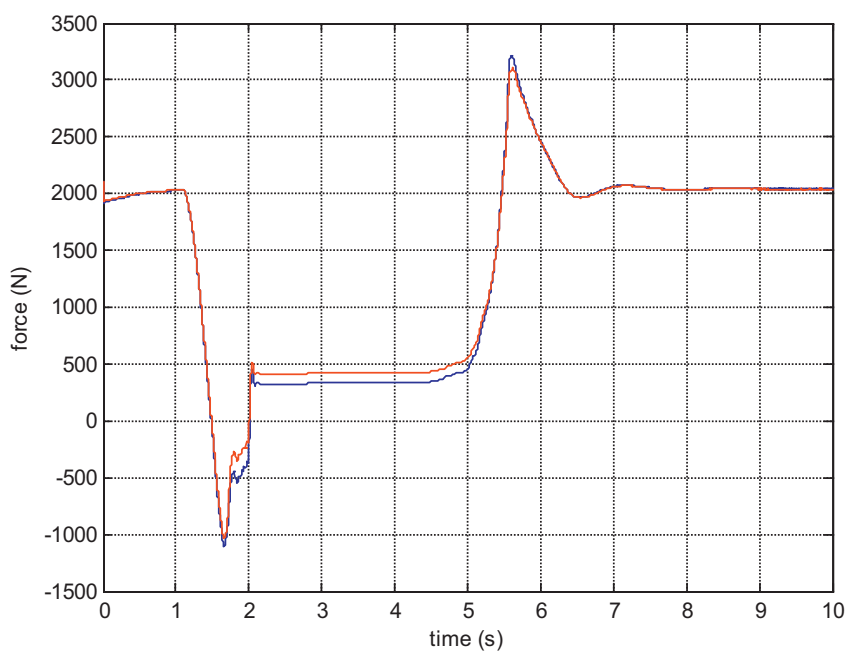

Fig. 15. Lateral force $Y_{r w}^{R}$.

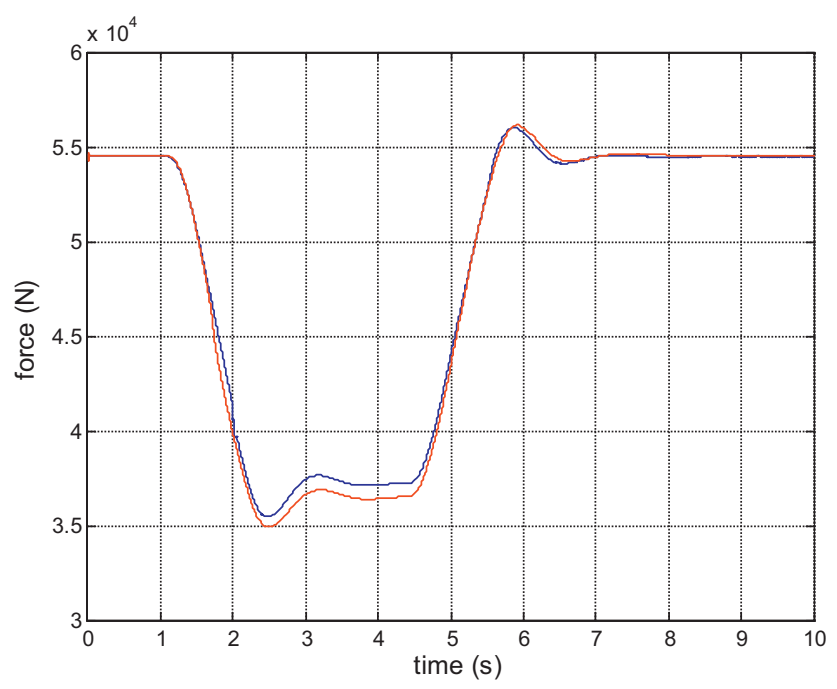

Fig. 16. Vertical force $Q_{r w}^{R}$.

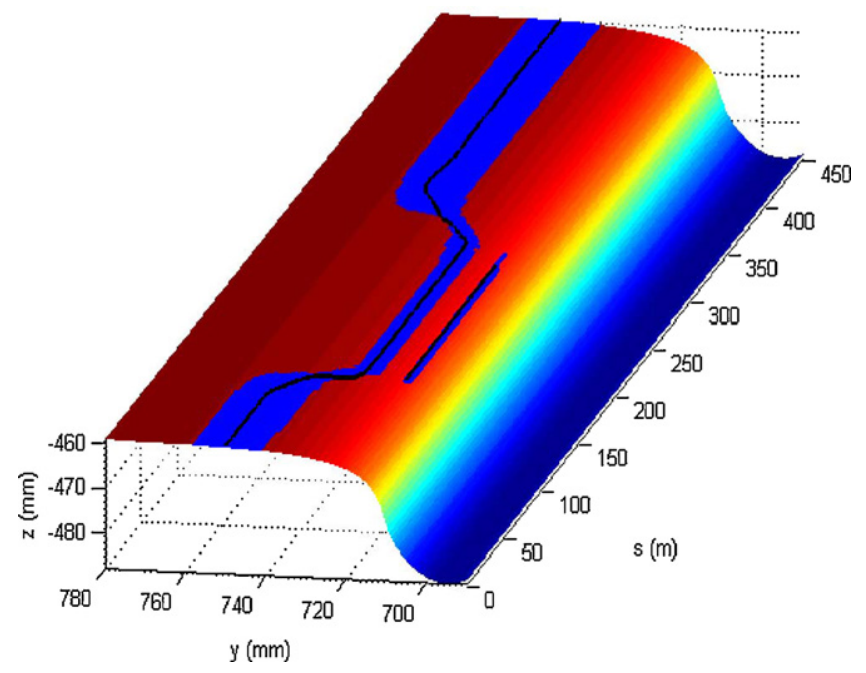

Fig. 17. Section of contact area $A_{l w c}$.

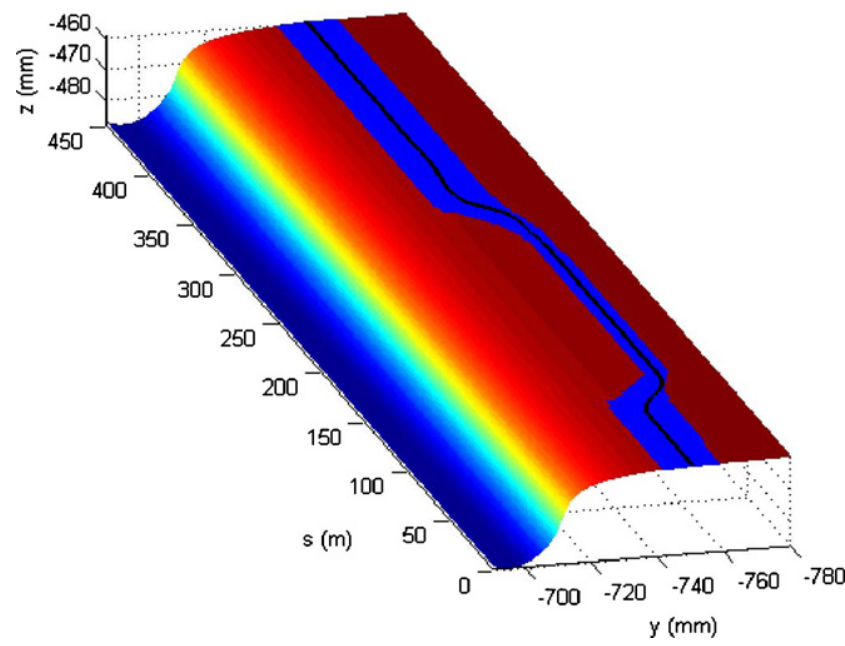

Fig. 18. Section of contact area $A_{r w c}$.

Simulation results show good agreement between the Matlab model and the Simpack model, both in terms of kinematic variables and contact forces.

In order to give an effective description of the motion of the contact areas during the simulation, a lateral section along the plane $y_{R} z_{R}$ of the areas $A_{w c}, A_{r C}$ was considered. Moreover, contact patches have been plotted on cylindrical surfaces generated by the wheel and rail profiles over a length as long as the distance traveled by the vehicle. By convention $A_{l w C}, A_{r w C}$ are the contact areas on the left and on the right wheel (Figs. 17 and 18) while $A_{l r c}, A_{r r c}$ are the contact areas on the left and on the right rail (Figs. 19 and 20).

The sections of contact area evaluated by the Matlab model are plotted in blue (For interpretation of the references to color in the text, the reader is referred to the web version of this article) while the contact points detected by the Simpack model are plotted in black. It is interesting to note that during curving a second contact point appears on the left wheel and rail (the track turns to right). Consequently, while the Simpack model detects two distinct contact points, the contact areas evaluated by the Matlab model consist of two disjointed parts. Also in this case the agreement between the results obtained by the Matlab model and the Simpack model is generally very good.

In conclusion the accuracy of the Matlab model turns out to be comparable with that of the Simpack model; moreover the quasi- 


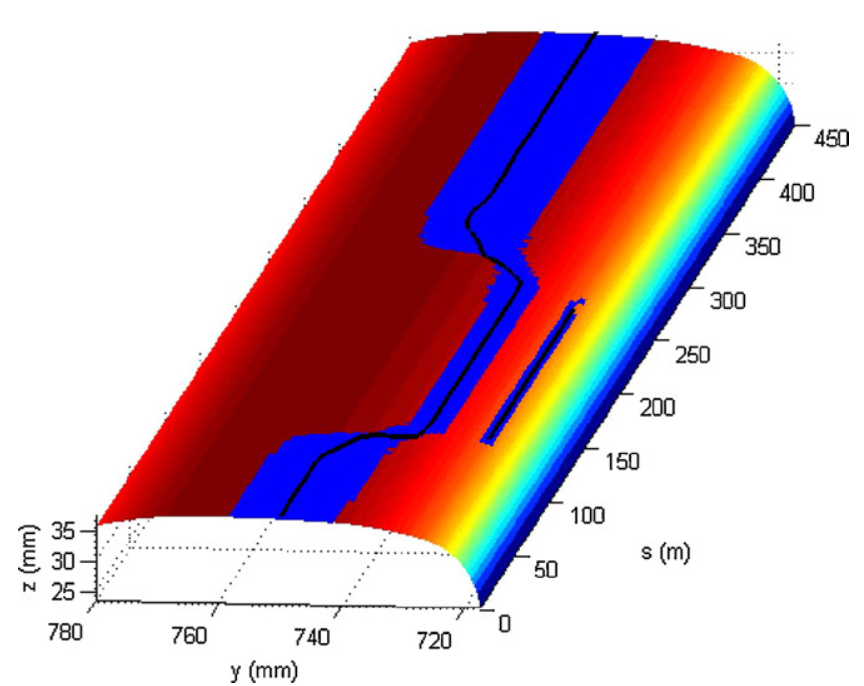

Fig. 19. Section of contact area $A_{\text {lrc }}$.

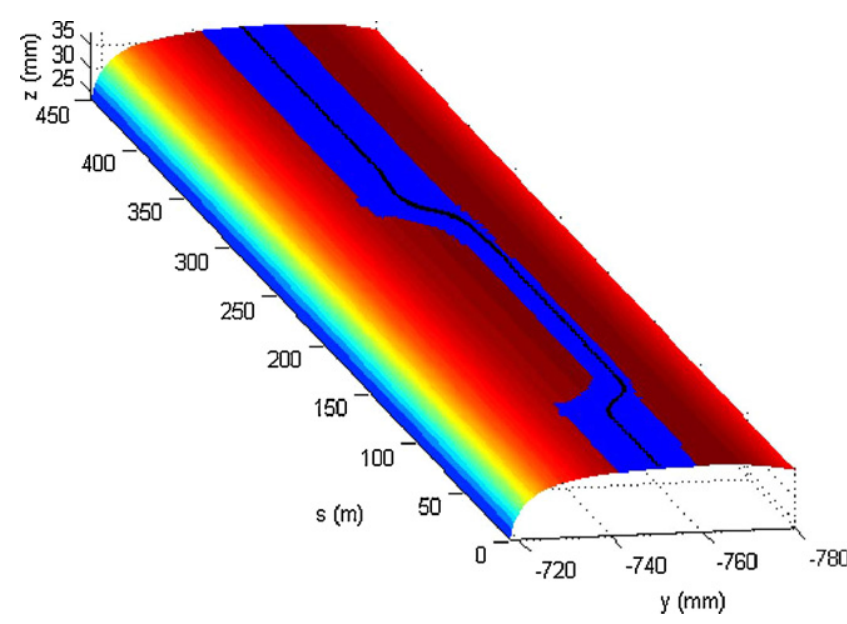

Fig. 20. Section of contact area $A_{\text {rrc }}$.

total absence of numerical noise highlights the robustness and the stability of the new differential contact model.

\section{Conclusion and perspective}

The performance of the Matlab model turned out to be good both in terms of output accuracy (kinematic variables, contact forces and contact patch) and in terms of numerical efficiency (performance of the numerical algorithms and time consumption), thus satisfying the main objectives of the study.
In regards to further developments, a fully 3D multibody model of the Manchester Wagon will be considered in the future. This type of model allows a complete description of the vehicle dynamics but obviously involves an increase in DOFs and of the number of wheel-rail contact pairs.

Further optimization of the differential contact model is also planned for the future. Such improvements will specifically target the FEM techniques used to discretize the contact problem. New mesh generation algorithms and suitable nonlinear shape functions will also be examined. While these techniques will ensure greater accuracy, they will also increase computational load and memory consumption. As such, final development of the contact model into programming environments like $\mathrm{C} / \mathrm{C}++$ and FORTRAN will be considered in order to reduce computation time.

\section{References}

[1] A.A. Shabana, J.R. Sany, Nonlinear Dynamics 24 (2001) 183-204.

[2] S. Iwinicki, Fatigue and Fracture of Engineering Materials and Structures 26 (2003) 887-900.

[3] J. Pombo, J. Ambrosio, International Journal of Vehicle Systems Modelling and Testing 1 (2005) 79-105.

[4] J.J. Kalker, Three-Dimensional Elastic Bodies in Rolling Contact, Kluwer Academic Publishers, Dordrecht, Netherlands, 1990.

[5] R.V. Dukkipati, J.R. Amyot, Computer Aided Simulation in Railway Dynamics, Dekker, New York, 1988.

[6] O. Polach, Wear 258 (2005) 992-1000.

[7] A.A. Shabana, K.E. Zaazaa, J.L. Escalona, J.L. Sany, Journal of Sound and Vibration 269 (2004) 295-325.

[8] S. Falomi, M. Malvezzi, E. Meli, A. Rindi, Multibody System Dynamics 20 (4) (2008) 327-358.

[9] K.L. Johnson, Contact Mechanics, Cambridge University Press, Cambridge, England, 1985.

[10] N. Kikuchi, J.T. Oden, Contact Problems in Elasticity, SIAM Studies in Applied Mathematics, Philadelphia, PA, 1988.

[11] P. Wriggers, Computational Contact Mechanics, John Wiley \& Sons, Hoboken, NJ, 2002.

[12] O. Zienkiewicz, The Finite Element Method in Engineering Science, McGrawHill, New York, 1988.

[13] A. Klarbring, Computer Methods in Applied Mechanics and Engineering 58 (1986) 175-200.

[14] S. Iwinicki, The Manchester Benchmarks for Rail Vehicle Simulators, Swets \& Zeitlinger, Lisse, Netherlands, 1999.

[15] C. Esveld, Modern Railway Track, Delft University of Technology, Delft, Netherlands, 2001.

[16] G. Dhatt, G. Touzot, The Finite Element Method Displayed, John Wiley \& Sons, Hoboken, NJ, 1984.

[17] G. Vicuna, Organizzazione e Tecnica Ferroviaria, Ed. CIFI, Roma, Italy, 1986.

[18] R.S. Dembo, S.C. Eisenstat, T. Steihaug, SIAM Journal of Numerical Analysis 19 (1982) 400-408.

[19] C.T. Kelley, Iterative Methods for Linear and Nonlinear Equations, SIAM, Philadelphia, PA, 1995.

[20] Y. Saad, Iterative Methods for Sparse Linear Systems, SIAM, Philadelphia, PA, 2003.

[21] J. Nocedal, S.J. Wright, Numerical Optimization, Springer Series in Operation Research, Berlin, Germany, 1999.

[22] L.F. Shampine, M.W. Reichelt, The MATLAB ODE Suite, SIAM Journal on Scientific Computing 18 (1997) 1-22.

[23] S. Bellavia, B. Morini, SIAM Journal on Scientific Computing 23 (2001) 940960. 